Bull. Austral. Math. Soc.

VoL. $72(2005) \quad[45-52]$

\title{
A REMARKABLE CONTINUED FRACTION
}

\section{David ANgell and Michael D. Hirschhorn}

We study a particular oscillating continued fraction, and find its two limit points.

Let $\left\{a_{k}\right\}_{k \in \mathbb{N}},\left\{b_{k}\right\}_{k \in \mathbb{N}}$ be sequences of positive integers, and consider the continued fraction

$$
\frac{b_{1}}{a_{1}}+\frac{b_{2}}{a_{2}}+\frac{b_{3}}{a_{3}}+\cdots
$$

The convergents $p_{k} / q_{k}$ to this continued fraction are defined recursively,

$$
p_{k}=a_{k} p_{k-1}+b_{k} p_{k-2}, \quad q_{k}=a_{k} q_{k-1}+b_{k} q_{k-2},
$$

with the initial conditions $p_{-1}=1, p_{0}=0, q_{-1}=0, q_{0}=1$. It is then easy to show that

$$
\frac{b_{1}}{a_{1}}+\frac{b_{2}}{a_{2}}+\frac{b_{3}}{a_{3}}+\cdots \frac{b_{k}}{a_{k}}=\frac{p_{k}}{q_{k}}
$$

that $q_{k}$ increases without limit as $k \rightarrow \infty$, and that

$$
\frac{p_{k}}{q_{k}}-\frac{p_{k-1}}{q_{k-1}}=\frac{(-1)^{k} b_{k} b_{k-1} \cdots b_{1}}{q_{k} q_{k-1}} .
$$

Since $a_{k}$ and $b_{k}$ are positive, the convergent

$$
\frac{p_{k}}{q_{k}}=\frac{a_{k} p_{k-1}+b_{k} p_{k-2}}{a_{k} q_{k-1}+b_{k} q_{k-2}}
$$

lies strictly between $p_{k-1} / q_{k-1}$ and $p_{k-2} / q_{k-2}$; it is easy to check that the second convergent is less than the first, and so

$$
\frac{p_{2}}{q_{2}}<\frac{p_{4}}{q_{4}}<\frac{p_{6}}{q_{6}}<\cdots<\frac{p_{5}}{q_{5}}<\frac{p_{3}}{q_{3}}<\frac{p_{1}}{q_{1}} .
$$

It follows immediately that

$$
\lim _{k \rightarrow \infty} \frac{p_{2 k}}{q_{2 k}} \text { and } \lim _{k \rightarrow \infty} \frac{p_{2 k-1}}{q_{2 k-1}}
$$

Received 7th February, 2005

Copyright Clearance Centre, Inc. Serial-fee code: 0004-9727/05 $\$ A 2.00+0.00$. 
both exist. If every $b_{k}$ is 1 , then from (2) we have

$$
\lim _{k \rightarrow \infty}\left(\frac{p_{k}}{q_{k}}-\frac{p_{k-1}}{q_{k-1}}\right)=0
$$

and so $p_{2 k} / q_{2 k}$ and $p_{2 k-1} / q_{2 k-1}$ approach a common limit. In the general case, however, this need not be true. The continued fraction (1) is said to converge if the two limits (3) are the same, and to oscillate if not. A simple sufficient condition for convergence is that $a_{k} \geqslant b_{k}$ for all large $k$, and a necessary and sufficient condition may be found in [1]: the continued fraction (1) converges if and only if at least one of the series

$$
\frac{a_{1}}{b_{1}}+\frac{a_{3} b_{2}}{b_{3} b_{1}}+\frac{a_{5} b_{4} b_{2}}{b_{5} b_{3} b_{1}}+\frac{a_{7} b_{6} b_{4} b_{2}}{b_{7} b_{5} b_{3} b_{1}}+\cdots
$$

and

$$
\frac{a_{2} b_{1}}{b_{2}}+\frac{a_{4} b_{3} b_{1}}{b_{4} b_{2}}+\frac{a_{6} b_{5} b_{3} b_{1}}{b_{6} b_{4} b_{2}}+\cdots
$$

diverges. From this result it is easy to see that the continued fraction given by $a_{k}=1$, $b_{k}=2^{k}$

$$
\frac{2}{1}+\frac{4}{1}+\frac{8}{1}+\frac{16}{1}+\cdots
$$

oscillates, but it seems difficult to evaluate the two limit points of $p_{k} / q_{k}$. We made the surprising discovery that if we tweak the $b_{k}$ just a little, we obtain a tractable problem. Indeed we prove

THEOREM. For the continued fraction given by $a_{k}=1, b_{k}=2^{k}+2$,

$$
\frac{4}{1}+\frac{6}{1}+\frac{10}{1}+\frac{18}{1}+\cdots \frac{2^{k}+2}{1}+\cdots
$$

we have

$$
\lim _{k \rightarrow \infty} \frac{p_{2 k}}{q_{2 k}}=1 \text { and } \lim _{k \rightarrow \infty} \frac{p_{2 k-1}}{q_{2 k-1}}=2
$$

Proof: We have

$$
\begin{aligned}
& p_{0}=0, \quad p_{1}=4, \quad p_{k}=p_{k-1}+\left(2^{k}+2\right) p_{k-2} \\
& q_{0}=1, \quad q_{1}=1, \quad q_{k}=q_{k-1}+\left(2^{k}+2\right) q_{k-2} .
\end{aligned}
$$

The first few $p_{k}, q_{k}$ are given by the table

$$
\begin{array}{cccccccc}
k & 0 & 1 & 2 & 3 & 4 & 5 & \ldots \\
p_{k} & 0 & 4 & 4 & 44 & 116 & 1612 & \ldots \\
q_{k} & 1 & 1 & 7 & 17 & 143 & 721 & \ldots
\end{array}
$$


It is not hard to show from (4) that

$$
p_{k}=\left(2^{k}+2^{k-1}+5\right) p_{k-2}-\left(2^{2 k-3}+2^{k}+2^{k-1}+4\right) p_{k-4},
$$

and, of course, the same recurrence holds for the $\left\{q_{k}\right\}$. Thus,

$$
\begin{aligned}
p_{k} & =p_{k-1}+\left(2^{k}+2\right) p_{k-2} \\
& =p_{k-2}+\left(2^{k-1}+2\right) p_{k-3}+\left(2^{k}+2\right) p_{k-2} \\
& =\left(2^{k}+3\right) p_{k-2}+\left(2^{k-1}+2\right) p_{k-3} \\
& =\left(2^{k}+3\right) p_{k-2}+\left(2^{k-1}+2\right)\left(p_{k-2}-\left(2^{k-2}+2\right) p_{k-4}\right) \\
& =\left(2^{k}+2^{k-1}+5\right) p_{k-2}-\left(2^{2 k-3}+2^{k}+2^{k-1}+4\right) p_{k-4}
\end{aligned}
$$

So we have

$$
p_{2 k}=\left(2^{2 k}+2^{2 k-1}+5\right) p_{2 k-2}-\left(2^{4 k-3}+2^{2 k}+2^{2 k-1}+4\right) p_{2 k-4}
$$

and

$$
p_{2 k+1}=\left(2^{2 k+1}+2^{2 k}+5\right) p_{2 k-1}-\left(2^{4 k-1}+2^{2 k+1}+2^{2 k}+4\right) p_{2 k-3}
$$

and the same recurrences hold for $q_{2 k}, q_{2 k+1}$ respectively.

We now define

$$
\begin{aligned}
& P_{e}(x)=\sum_{k \geqslant 0} p_{2 k} x^{k}, \quad P_{o}(x)=\sum_{k \geqslant 0} p_{2 k+1} x^{k}, \\
& Q_{e}(x)=\sum_{k \geqslant 0} q_{2 k} x^{k}, \quad Q_{o}(x)=\sum_{k \geqslant 0} q_{2 k+1} x^{k} .
\end{aligned}
$$

From (6) it follows that

(8) $P_{e}(x)-5 x P_{e}(x)+4 x^{2} P_{e}(x)-6 x P_{e}(4 x)+24 x^{2} P_{e}(4 x)+32 x^{2} P_{e}(16 x)$

$$
=p_{0}+\left(p_{2}-11 p_{0}\right) x=4 x,
$$

while from (7)

(9) $P_{\circ}(x)-5 x P_{o}(x)+4 x^{2} P_{o}(x)-12 x P_{o}(4 x)+48 x^{2} P_{o}(4 x)+128 x^{2} P_{o}(16 x)$

$$
=p_{1}+\left(p_{3}-17 p_{1}\right) x=4-24 x \text {. }
$$

Similar relations hold for $Q_{e}(x), Q_{o}(x)$ respectively. 
(8) and (9) can be written

$$
\begin{aligned}
& P_{e}(x)=\frac{4 x}{(1-x)(1-4 x)}+\frac{6 x}{(1-x)} P_{e}(4 x)-\frac{32 x^{2}}{(1-x)(1-4 x)} P_{e}(16 x), \\
& P_{o}(x)=\frac{4-24 x}{(1-x)(1-4 x)}+\frac{12 x}{(1-x)} P_{o}(4 x)-\frac{128 x^{2}}{(1-x)(1-4 x)} P_{o}(16 x) .
\end{aligned}
$$

Iteration of (10) leads to to the following, which we prove by induction.

$$
\begin{aligned}
P_{e}(x)=\sum_{k=0}^{n} \frac{\left(2^{k^{2}+3 k+3}-2^{k^{2}+2 k+2}\right) x^{k+1}}{(1-x)(1-4 x) \cdots\left(1-4^{k+1} x\right)} \\
\quad+\frac{\left(2^{n^{2}+3 n+3}-2^{n^{2}+2 n+1}\right) x^{n+1}}{(1-x) \cdots\left(1-4^{n} x\right)} P_{e}\left(4^{n+1} x\right) \\
\quad-\frac{\left(2^{n^{2}+5 n+6}-2^{n^{2}+4 n+5}\right) x^{n+2}}{(1-x)(1-4 x) \cdots\left(1-4^{n+1} x\right)} P_{e}\left(4^{n+2} x\right) .
\end{aligned}
$$

First, (12) is true for $n=0$ by (10). Also, if we put $4^{n+1} x$ for $x$ in (10), we obtain

$$
\begin{aligned}
P_{e}\left(4^{n+1} x\right)=\frac{4^{n+2} x}{\left(1-4^{n+1} x\right)\left(1-4^{n+2} x\right)} & +\frac{6 \times 4^{n+1} x}{\left(1-4^{n+1} x\right)} P_{e}\left(4^{n+2} x\right) \\
& -\frac{32 \times 4^{2 n+2} x^{2}}{\left(1-4^{n+1} x\right)\left(1-4^{n+2} x\right)} P_{e}\left(4^{n+3} x\right)
\end{aligned}
$$

If we suppose (12) true for some $n \geqslant 0$, and we substitute (13) into (12), we obtain

$$
\begin{gathered}
P_{e}(x)=\sum_{k=0}^{n} \frac{\left(2^{k^{2}+3 k+3}-2^{k^{2}+2 k+2}\right) x^{k+1}}{(1-x)(1-4 x) \cdots\left(1-4^{k+1} x\right)} \\
+\frac{\left(2^{n^{2}+3 n+3}-2^{n^{2}+2 n+1}\right) x^{n+1}}{(1-x) \cdots\left(1-4^{n} x\right)} \\
\quad \times\left\{\frac{4^{n+2} x}{\left(1-4^{n+1} x\right)\left(1-4^{n+2} x\right)}+\frac{6 \times 4^{n+1} x}{\left(1-4^{n+1} x\right)} P_{e}\left(4^{n+2} x\right)\right. \\
\left.-\frac{32 \times 4^{2 n+2} x^{2}}{\left(1-4^{n+1} x\right)\left(1-4^{n+2} x\right)} P_{e}\left(4^{n+3} x\right)\right\} \\
-\frac{\left(2^{n^{2}+5 n+6}-2^{n^{2}+4 n+5}\right) x^{n+2}}{(1-x)(1-4 x) \cdots\left(1-4^{n+1} x\right)} P_{e}\left(4^{n+2} x\right) \\
=\sum_{k=0}^{n+1} \frac{\left(2^{k^{2}+3 k+3}-2^{k^{2}+2 k+2}\right) x^{k+1}}{(1-x)(1-4 x) \cdots\left(1-4^{k+1} x\right)}
\end{gathered}
$$




$$
\begin{aligned}
& +\frac{\left(2^{(n+1)^{2}+3(n+1)+3}-2^{(n+1)^{2}+2(n+1)+1}\right) x^{n+2}}{(1-x)(1-4 x) \cdots\left(1-4^{n+1} x\right)} P_{e}\left(4^{n+2} x\right) \\
& -\frac{\left(2^{(n+1)^{2}+5(n+1)+6}-2^{(n+1)^{2}+4(n+1)+5}\right) x^{n+3}}{(1-x)(1-4 x) \cdots\left(1-4^{n+2}\right) x} P_{e}\left(4^{n+3} x\right) .
\end{aligned}
$$

Here we have used the facts that

$$
\begin{aligned}
4^{n+2}\left(2^{n^{2}+3 n+3}-2^{n^{2}+2 n+1}\right) & =2^{(n+1)^{2}+3(n+1)+3}-2^{(n+1)^{2}+2(n+1)+2} \\
6 \times 4^{n+1}\left(2^{n^{2}+3 n+3}-2^{n^{2}+2 n+1}\right) & -\left(2^{n^{2}+5 n+6}-2^{n^{2}+4 n+5}\right) \\
& =2^{(n+1)^{2}+3(n+1)+3}-2^{(n+1)^{2}+2(n+1)+1}, \\
32 \times 4^{2 n+2}\left(2^{n^{2}+3 n+3}-2^{n^{2}+2 n+1}\right) & =2^{(n+1)^{2}+5(n+1)+6}-2^{(n+1)^{2}+4(n+1)+5} .
\end{aligned}
$$

That is, (12) is true for $n+1$. So (12) is true for $n \geq 0$ by induction. If we let $n \rightarrow \infty$ we find

$$
P_{e}(x)=\sum_{k \geqslant 0} \frac{\left(2^{k^{2}+k+1}-2^{k^{2}+1}\right) x^{k}}{(1-x) \cdots\left(1-4^{k} x\right)}
$$

In the same way, we can show that

$$
\begin{aligned}
& P_{o}(x)=\sum_{k \geqslant 0} \frac{\left(2^{k^{2}+2 k+1}-2^{k^{2}+k}\right)\left(4-24 \times 4^{k} x\right) x^{k}}{(1-x)(1-4 x) \cdots\left(1-4^{k+1} x\right)} \\
& Q_{e}(x)=\sum_{k \geqslant 0} \frac{\left(2^{k^{2}+k+1}-2^{k^{2}}\right) x^{k}}{(1-x) \cdots\left(1-4^{k} x\right)} \\
& Q_{o}(x)=\sum_{k \geqslant 0} \frac{\left(2^{k^{2}+2 k+1}-2^{k^{2}+k}\right) x^{k}}{(1-x)(1-4 x) \cdots\left(1-4^{k+1} x\right)}
\end{aligned}
$$

It is not hard to show that

$$
\begin{aligned}
& P_{o}(x)=\sum_{k \geqslant 0} \frac{\left(3 \times 2^{k^{2}+2 k+1}-2 \times 2^{k^{2}+k}\right) x^{k}}{(1-x) \cdots\left(1-4^{k} x\right)} \\
& Q_{o}(x)=\sum_{k \geqslant 0} \frac{\left(3 \times 2^{k^{2}+2 k}-2 \times 2^{k^{2}+k}\right) x^{k}}{(1-x) \cdots\left(1-4^{k} x\right)} .
\end{aligned}
$$


For instance,

$$
\begin{aligned}
P_{o}(x) & =\sum_{k \geqslant 0} \frac{\left(2^{k^{2}+2 k+1}-2^{k^{2}+k}\right)\left(6\left(1-4^{k+1} x\right)-2\right) x^{k}}{(1-x)(1-4 x) \cdots\left(1-4^{k+1} x\right)} \\
& =\sum_{k \geqslant 0} \frac{6\left(2^{k^{2}+2 k+1}-2^{k^{2}+k}\right) x^{k}}{(1-x) \cdots\left(1-4^{k} x\right)}-\sum_{k \geqslant 0} \frac{2\left(2^{k^{2}+2 k+1}-2^{k^{2}+k}\right) x^{k}}{(1-x) \cdots\left(1-4^{k} x\right)} \cdot\left(1+\frac{4^{k+1} x}{1-4^{k+1} x}\right) \\
& =\sum_{k \geqslant 0} \frac{4\left(2^{k^{2}+2 k+1}-2^{k^{2}+k}\right) x^{k}}{(1-x) \cdots\left(1-4^{k} x\right)}-\sum_{k \geqslant 0} \frac{\left(2^{k^{2}+4 k+4}-2^{k^{2}+3 k+3}\right) x^{k+1}}{(1-x) \cdots\left(1-4^{k+1} x\right)} \\
& =\sum_{k \geqslant 0} \frac{4\left(2^{k^{2}+2 k+1}-2^{k^{2}+k}\right) x^{k}}{(1-x) \cdots\left(1-4^{k} x\right)}-\sum_{k \geqslant 1} \frac{\left(2^{k^{2}+2 k+1}-2^{k^{2}+k+1}\right) x^{k}}{(1-x) \cdots\left(1-4^{k} x\right)} \\
& =\sum_{k \geqslant 0} \frac{\left(3 \times 2^{k^{2}+2 k+1}-2^{k^{2}+k+1}\right) x^{k}}{(1-x) \cdots\left(1-4^{k} x\right)} .
\end{aligned}
$$

Now let

$$
\begin{aligned}
& A(x)=\sum_{k \geqslant 0} \frac{2^{k^{2}+k} x^{k}}{(1-x) \cdots\left(1-4^{k} x\right)}=\sum_{k \geqslant 0} a_{k} x^{k}, \\
& B(x)=\sum_{k \geqslant 0} \frac{2^{k^{2}} x^{k}}{(1-x) \cdots\left(1-4^{k} x\right)}=\sum_{k \geqslant 0} b_{k} x^{k}, \\
& C(x)=\sum_{k \geqslant 0} \frac{3 \times 2^{k^{2}+2 k} x^{k}}{(1-x) \cdots\left(1-4^{k} x\right)}=\sum_{k \geqslant 0} c_{k} x^{k} .
\end{aligned}
$$

Then

$$
\begin{array}{ll}
P_{e}(x)=2 A(x)-2 B(x), & P_{o}(x)=2 C(x)-2 A(x) \\
Q_{e}(x)=2 A(x)-B(x), & Q_{o}(x)=C(x)-2 A(x)
\end{array}
$$

from which it follows that

$$
p_{2 k}=2 a_{k}-2 b_{k}, \quad p_{2 k+1}=2 c_{k}-2 a_{k}, \quad q_{2 k}=2 a_{k}-b_{k}, \quad q_{2 k+1}=c_{k}-2 a_{k}
$$

We have

$$
\begin{aligned}
& A(x)=\frac{1}{1-x}+\frac{4 x}{1-x} A(4 x), \quad B(x)=\frac{1}{1-x}+\frac{2 x}{1-x} B(4 x), \\
& C(x)=\frac{3}{1-x}+\frac{8 x}{1-x} C(4 x)
\end{aligned}
$$


or,

$$
\begin{aligned}
& A(x)=1+x A(x)+4 x A(4 x), \\
& B(x)=1+x B(x)+2 x B(4 x), \\
& C(x)=3+x C(x)+8 x C(4 x) .
\end{aligned}
$$

It follows that

$$
\begin{array}{ll}
a_{0}=1, & a_{k}=a_{k-1}+4^{k} a_{k-1}=\left(4^{k}+1\right) a_{k-1}, \\
b_{0}=1, & b_{k-1}+2 \times 4^{k-1} b_{k-1}=\left(2 \times 4^{k-1}+1\right) b_{k-1}, \\
c_{0}=3, & c_{k}=c_{k-1}+8 \times 4^{k-1} c_{k-1}=\left(2 \times 4^{k}+1\right) c_{k-1} .
\end{array}
$$

It follows that

$$
\begin{aligned}
& a_{k}=(4+1)\left(4^{2}+1\right) \cdots\left(4^{k}+1\right), \\
& b_{k}=(2+1)(2 \times 4+1) \cdots\left(2 \times 4^{k-1}+1\right), \\
& c_{k}=3(2 \times 4+1)\left(2 \times 4^{2}+1\right) \cdots\left(2 \times 4^{k}+1\right) .
\end{aligned}
$$

Note that $c_{k}=b_{k+1}$, so (19) becomes

(23) $p_{2 k}=2 a_{k}-2 b_{k}, \quad p_{2 k+1}=2 b_{k+1}-2 a_{k}, \quad q_{2 k}=2 a_{k}-b_{k}, \quad q_{2 k+1}=b_{k+1}-2 a_{k}$.

Observe the table

$\begin{array}{cccccccc}k & 0 & 1 & 2 & 3 & 4 & 5 & \ldots \\ p_{k} & 0 & 4 & 4 & 44 & 116 & 1612 & \ldots \\ q_{k} & 1 & 1 & 7 & 17 & 143 & 721 & \ldots \\ a_{k} & 1 & 5 & 85 & 5525 & 1419925 & 1455423125 & \ldots \\ b_{k} & 1 & 3 & 27 & 891 & 114939 & 58963707 & \ldots\end{array}$

Now,

$$
\frac{b_{k}}{a_{k}}=\frac{(2+1)}{(4+1)} \cdots \frac{\left(2 \times 4^{k-1}+1\right)}{\left(4^{k}+1\right)} \leqslant\left(\frac{3}{5}\right)^{k}
$$

and

$$
\frac{a_{k}}{b_{k+1}}=\frac{1}{(2+1)} \frac{(4+1)}{(2 \times 4+1)} \cdots \frac{\left(4^{k}+1\right)}{\left(2 \times 4^{k}+1\right)} \leqslant \frac{1}{3}\left(\frac{5}{9}\right)^{k} .
$$

It follows that

$$
1>\frac{p_{2 k}}{q_{2 k}}=1-\frac{b_{k} / a_{k}}{2-b_{k} / a_{k}} \geqslant 1-\frac{(3 / 5)^{k}}{2-(3 / 5)^{k}} \geqslant 1-\left(\frac{3}{5}\right)^{k}
$$

and

$$
2<\frac{p_{2 k+1}}{q_{2 k+1}}=2+\frac{2 a_{k} / b_{k+1}}{1-2 a_{k} / b_{k+1}} \leqslant 2+\frac{(2 / 3)(5 / 9)^{k}}{1-(2 / 3)(5 / 9)^{k}} \leqslant 2+2\left(\frac{5}{9}\right)^{k} .
$$

The result follows. 


\section{REFERENCES}

[1] G. Chrystal, Algebra (Chelsea Publishing Co., New York, 1964).

School of Mathematics

UNSW

Sydney NSW 2052

Australia 